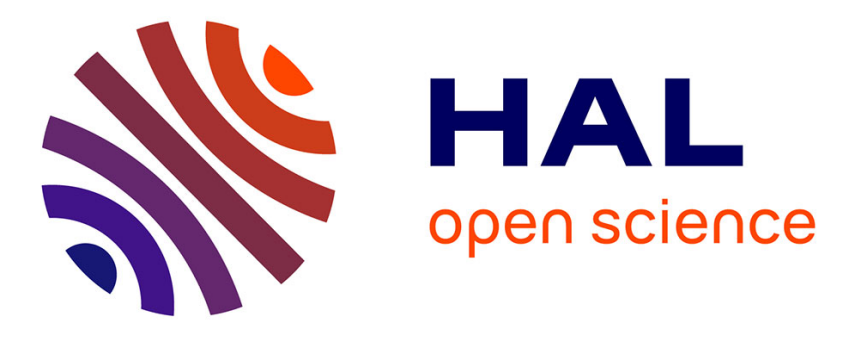

\title{
User mobility in a web-based communication system
}

Sivasothy Shanmugalingam, Noel Crespi, Paul Labrogere

\section{To cite this version:}

Sivasothy Shanmugalingam, Noel Crespi, Paul Labrogere. User mobility in a web-based communication system. 4th International Conference on Internet Multimedia Systems Architecture and Applications (IMSAA 2010), Dec 2010, Bangalore, India. pp.1-6, 10.1109/IMSAA.2010.5729395 . hal-01305754

\section{HAL Id: hal-01305754 \\ https://hal.science/hal-01305754}

Submitted on 21 Apr 2016

HAL is a multi-disciplinary open access archive for the deposit and dissemination of scientific research documents, whether they are published or not. The documents may come from teaching and research institutions in France or abroad, or from public or private research centers.
L'archive ouverte pluridisciplinaire HAL, est destinée au dépôt et à la diffusion de documents scientifiques de niveau recherche, publiés ou non, émanant des établissements d'enseignement et de recherche français ou étrangers, des laboratoires publics ou privés. 


\title{
User mobility in a Web-based communication system
}

\author{
Sivasothy Shanmugalingam ${ }^{1,2}$, Noel Crespi $^{2}$, and Paul Labrogere ${ }^{1}$ \\ ${ }^{1}$ Alcatel Lucent Bell Labs France, Centre de Villarceaux, 91620, Nozay, France \\ ${ }^{2}$ Institut Telecom, Telecom SudParis, 9 rue C.Fourier, 91011, Evry, France \\ sivasothy.shanmugalingam@alcatel-lucent.com,noel.crespi@it-sudparis.eu \\ paul.labrogere@alcatel-lucent.com
}

\begin{abstract}
To support user mobility across different devices, communication session continuity should be enabled at session initiation and during a session. The session continuity can be managed by either a user or dedicated network nodes such as back-to-back user agent (B2BUA) in IP Multimedia Subsystem (IMS) architecture. When network manages the session continuity, user experience is enriched because users do not need to know how to perform session transfer. However, in IMS architecture, three different network nodes (SIP forking proxy, SIP registrar and B2BUA) need to cooperate for session continuity. When single user (callee) logs in from different devices, it is very hard to achieve cooperation. In addition, these network nodes should be scalable when many users request this service simultaneously. This paper proposes a general architecture that supports the user mobility and how the general architecture can be realized into a Web-based communication system. Our initial analytical result shows that the usage of computing resources (e.g. $\mathrm{CPU}$ ) is reduced while supporting scalability, thanks to the simple Web client service infrastructure and Web Socket connections on top of HTTP.
\end{abstract}

Index Terms - Personal mobility, session mobility, Web based communication system

\section{INTRODUCTION}

$\mathrm{T}_{\mathrm{the}}^{\mathrm{h}}$ he Third Generation Partnership Project (3GPP) defines the IP Multimedia Subsystem (IMS) standards that separate the control layer from the service layer. IMS adopts Session Initiation Protocol (SIP) of RFC 3261 [14] for providing session-based services. Inheriting from SIP, IMS supports user mobility by two different approaches such as personal and session mobility. When a user logs in from different devices (i.e. multiple registrations), personal mobility is enabled at the initiation whereas session mobility is enabled during the communication.

The IMS architecture exhibits two technical challenges in offering a combined solution for personal and session mobility without user intervention. First, it does not define design guidelines for a harmonized coordination among the involved entities such as SIP registrar, and forking proxy [14] in the service control layer and SIP Application Server (SIP AS) in the service layer. Therefore, it hinders the implementation. Second, deployment of this service in IMS is a challenging task because of the scalability problem in the service layer and service control layer [24]. The solutions for the scalability problem require more computing resources and engineering efforts; effectiveness of the solutions remains a question.

In this paper, our contribution is twofold. First, we propose a descriptive model that supports personal and session mobility. This descriptive model serves as a blueprint for the development of the communication system. Our descriptive model aims at a network box (cf. section IV) that manages and transfers sessions on behalf of the user. In addition, this network box should be easily integrated with other services, for example, an ambient intelligence system can provide information of a terminal that is near to the user, while the user is in move. Second, we propose a call flow that can be implemented in a Web-based communication system. We address the scalability issue by assigning a network box for each 'callee' and relying on HTTP.

The rest of the paper is organized as follows: Section II proposes a use case and the related technical requirements. We present our solution for personal and session mobility in Section III. Our solution is a descriptive model for the communication services. Therefore, in Section IV, we explain how our solution can be implemented in a Web-based communication system, followed by two call flows for two different scenarios i) network initiated session establishment and transfer, and ii) missed call situation. We discuss existing research work and an alternative solution in Section V. Finally, Section VI concludes the paper with suggestions for future work.

\section{USE CASE AND REQUIREMENTS}

We describe a use case, followed by requirements in this section. An elderly inhabitant is living in a smart home which can detect the context of inhabitant (e.g. location). He receives a video call on his TV screen while sitting in the living room. During the call, he feels sleepy and walks to the bedroom. Once he reaches the bedroom, he is able to continue his video call on his bedroom TV screen without any disruption or issuing any commands. Once he finishes the call, he issues a cancel command using remote button while in the bed. When a caretaker calls, the call is directed to the bedroom TV screen. His smart house has many TV screens installed in each room 
and has the ability to receive and make a video call. Figure 1 visually presents the above-described use case.

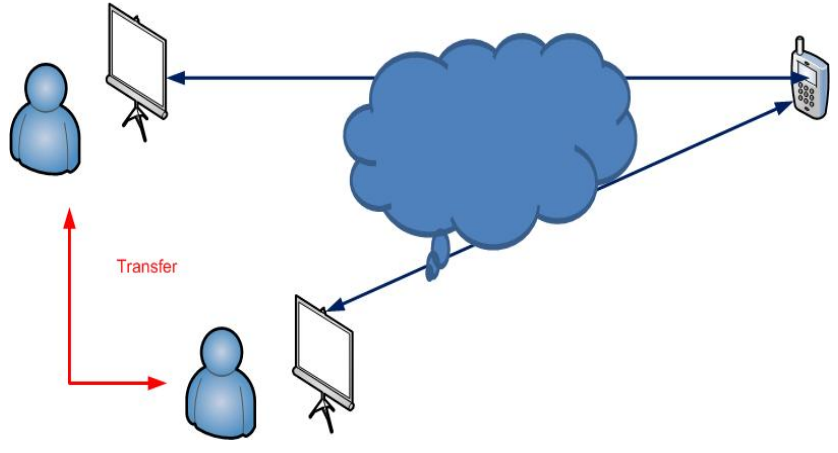

Figure 1 : Use case diagram.

From this use case, we derive the following requirements before designing an appropriate solution. For the clarity of the paper, we mention that the communication end points have audio/video processing capabilities and Web browser, connected to the Internet.

1. Multiple registrations: a user ('callee') should register in advance from different locations where the user moves around. This registration indicates the possible locations for transferring the calls by the network. When registering from different locations, the user uses the same username and password.

2. Network-initiated session transfer.

During the user's movements, after receiving ambient knowledge information, the network (or application) informs the user (here 'callee') about a new call. If the call is accepted by the user, media will be established. During the call, the user moves to a new communication end point. In this situation, the network informs the callee that call can be forwarded to your new location. Until the user accepts the call, media flow will not be disturbed. If the user accepts the call at the new end point, the network asks the caller to transfer the media to the new end point and terminates the session with the callee's previous end point. For our use case, network-initiated session transfer gives much user experience compared to user initiated.

3. Ambient knowledge (user context detection, distribution and derive high level knowledge). Our use case depends on context information of a person. The context information is location, e.g. which room the user is in. This low-level information should be derived to make a decision about which communication end point is near to the user. The framework that handles the ambient knowledge informs the communication system about the changes dynamically and provides high level information such as details of communication end points near to a user.

\section{OUR SOlution: DescriPTIVE Model}

Based on the requirements listed in the previous section, we define a general descriptive model, leveraging the descriptive model proposed in [1] which defines box, tunnel, slot and flow link. The proposal in [1] is initially developed for identifying correct media behavior by application servers. In this paper, we design to support the user mobility in the descriptive model as shown in Figure 2.

We identify three boxes: Caller Box, Callee Box and Network Box. A callee box and caller box are connected to a network box by signaling channel that is two way, first-in first-out (FIFO) and reliable. This model has many callee boxes and one caller box because of multiple callee registrations. Caller box expires once a call is finished, but callee boxes are in active stage until the callee closes the signaling channel. Figure 2 clearly shows media channel and signaling path before and after the session transfer.

Though our model is intuitive, we describe how our model manages a session. For our use case, session management is principally delegated to the network box which is an intermediary between caller and callee.

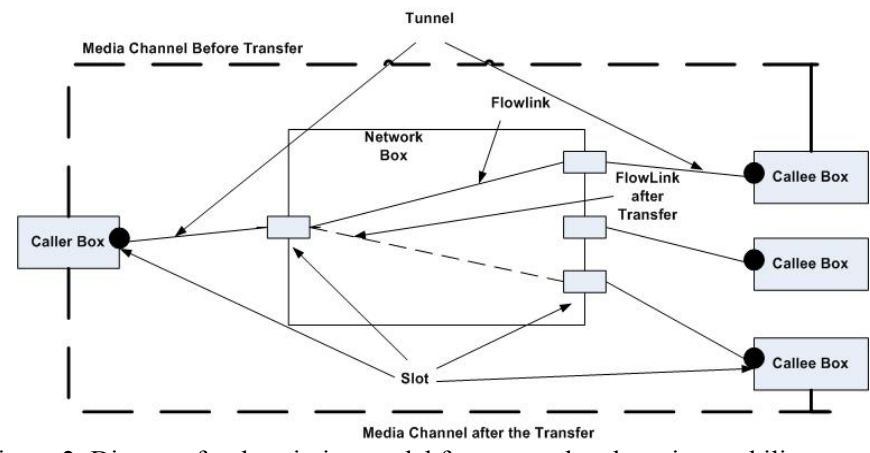

Figure 2: Diagram for descriptive model for personal and session mobility

For managing the session, the network box keeps information of callee/caller boxes such as IP address and IP port or TCP connection and dynamic attributes (such as closed, opening, opened and flowing) of media channel of each end point. Based on this information, logic in the network box performs session management at session initiation or during a session. In fact, the network box sets and updates the flow link with different callee boxes.

The network box connects two tunnels via a flow link. A tunnel is used to control a media channel and is established within a signaling channel. In other words, a signaling path is two tunnels and one flow link. Flow link is a software entity in the network box, representing a connection of two tunnels.

End points of a signaling path establish the media channel. One end of the media channel can be in four different states: closed, opening, opened and flowing, as modeled in [1]. Caller box and Callee box are software entities similar to user agents in SIP.

In Section IV, we describe how we realize the descriptive model in a Web-based communication system. Furthermore, we describe a new service which can be used in a missed call situation and can be developed based on the descriptive model.

\section{REALIZATION}

\section{A. Web-based communication system}

Figure 3 shows a simplified architecture for the Web-based 
communication system whose control plane is enabled by a Web application and whose media plane is empowered by a media server if both end points do not support the same media codec. This Web-based communication system is different from the existing communication systems because the caller uses a communication hyperlink provided by a callee to initiate a call.

If readers are interested in more details, cf. [21] [22].

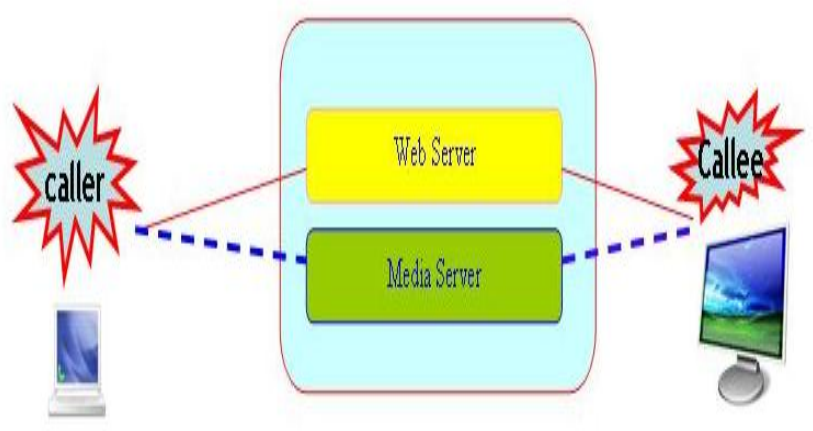

Figure 3: Simplified architecture of Web-based communication system.

This Web-based communication system is currently underexploited. For example, it is not widely used to facilitate mobility, but it has high potential to support network initiated session transfer. Now we describe how our descriptive model can be mapped into the Web-based communication system. The web server in Figure 3 hosts the network box and caller and callee box are hosted in the user's Web browser on demand from the Web server.

A Web Socket connection is proposed for bidirectional communication between Web server and browsers [2] [3]; it will increase the programming efficiency and reduce the latency for calls set up in the Web environment. Therefore, we use Web Socket connections for establishing the signaling channels between network box and callee box and network box and caller box.

The network box identifies users, based on TCP signaling channel established with the Web server and identity of the end users (e.g. any name, like a user account to the Web site). It is worth mentioning the approach we took to address the scalability. If one resource in a Web server is requested by many people at the same time, there is a performance issue in the Web server. But in our context every user has his own network box and each network box can be considered as a different resource. As such one network box will not receive many session requests from callers at a time.

Each network box is separate; it can be hosted separately or Web server can host many network boxes. Therefore, we consider vertical scalability where more resources (e.g. CPU, memory) will be added as many users are subscribed. Our approach will be effective even when many thousands of calls are made simultaneously compared to IMS solution where horizontal scalability is mainly needed and hard to develop.

\section{B. Network- initiated session transfer}

In this section, we discuss the functionality of the network box during the network-initiated session transfer, and present the corresponding call flow.

The network box makes decisions about which media channels should exist. In order to make the decision, the network box depends on a context enabler that will satisfy the requirement (2) as listed in Section II. In this paper, specification of the context enabler is out of scope, but a network box needs two functional interfaces such as:

A. Providing the location of the end user (IP address).

B. Providing the location of an end user during a session and if the user is in motion.

Separating the context enabler from the communication system serves to reduce the complexity. More information related to context (enabler) can be found in the C-CAST project website in which the context enabler provides user action, user situation and physical environment information [15][16].

In [19], M.E. Barachi et al propose a gateway for IMS and wireless sensor network (WSN) interworking. Data from the WSN provides context information about the user, but our solution is more focused on the communication service side.

For the session transfer, we decided to adopt a soft handoff to decrease the disruption time. Soft handoff means creating a session before breaking the former one.

We describe the call flow in the next two paragraphs. Callee $\mathrm{X}$ signs in to the Web server from the two communication end points identified by callee@diningroom and callee@bedroom in Figure 4. It means that both end points open a Web Socket connection with the Web server. We did not show the Web Socket connection opening and closing in Fig 4. Once the caller establishes a call (shown as CLICK message in Figure 4), the network box checks the context of the callee with the context enabler. The network box forwards the call to the right communication end point if the particular callee signs in from that end point. In Figure 4, network box sends the open message to Callee@DiningRoom. In Figure 4, CLICK and ACCEPT are two HTTP messages (request and response).

After the ACCEPT message, caller establishes a Web socket connection that is not shown in Figure 4. Other messages such as open, select, describe are defined in [1] and are routed via a Web socket connection. Session establishment is shown in Fig 4.

Figure 4 also shows the call flow for the session mobility; network box sends the open message to Callee@Bedroom when it receives the information of changed context. If callee accepts the session at the bedroom, network box sends a close message to the old location (dining room) and media description (describe (desc3)) to the caller. Then, the session is continued. More importantly, session setup time in this call flow during the movement is reduced because the network box is able to memorize the session description of caller and to send it to the new callee box. See the open (medium,desc2) message sent to callee@bedroom. Desc2 is received by the network box when caller initially starts the session.

Some messages (describe and select) are unilateral in Fig 4. 
Interested readers please refer to [1].

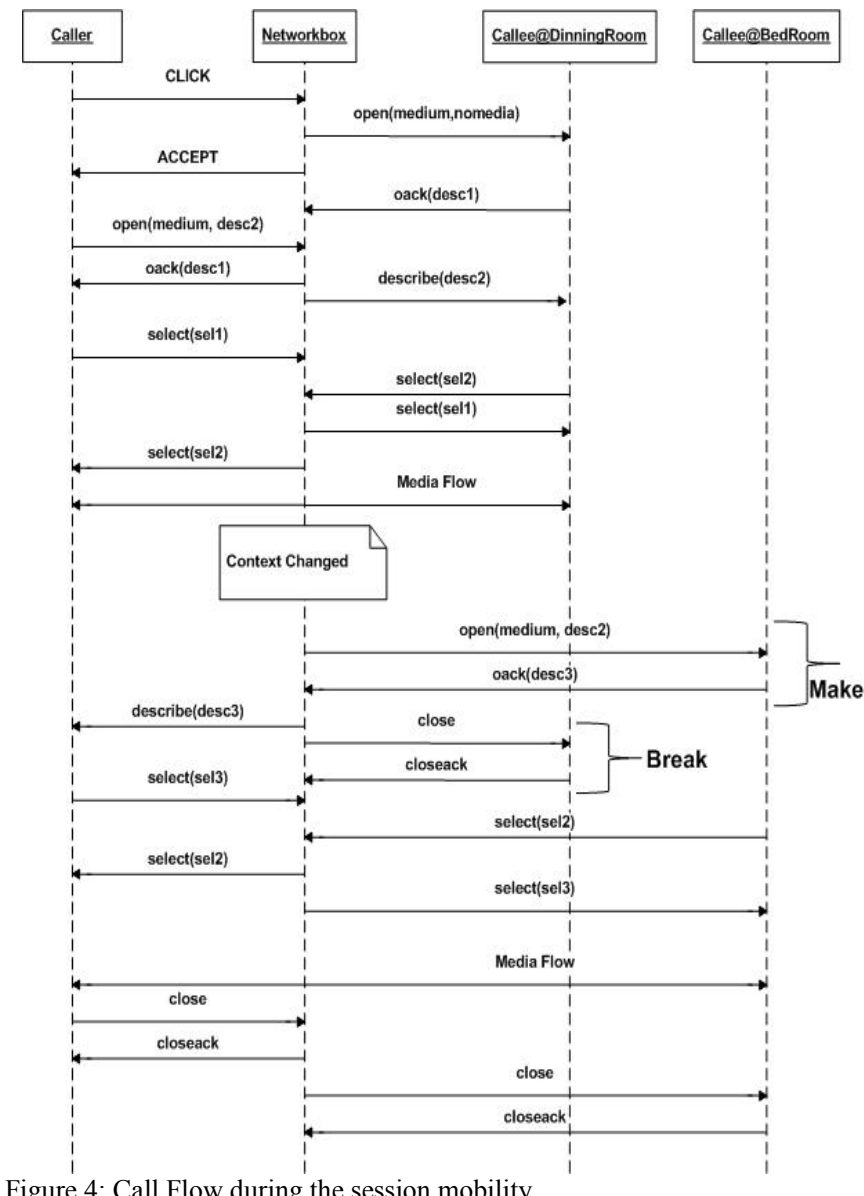

Figure 4: Call Flow during the session mobility

\section{Phenomenon of missed call}

In the case that user is not near to any end point, when a new call arrives, it will be missed because the user is not reachable. In this case, the caller should be placed to video/voice message service. During the voice/video recording, if the user becomes reachable, he will be notified that one caller is forwarded to the messaging services and wants to communicate. In this situation, if the callee is willing to communicate, the call will be established. We refer this use case as a session-based service composition.

This new use case is realized based on our descriptive model because voice mail server is an end point like callee, and network box can carry out specific logic. Based on this idea, we develop the call flow for this new use case as shown in Figure 5. We assume that Figure 5 has two kinds of call flow where the thick lines show the session with voice mail server and the dotted lines show update of the session for the voice communication between caller and callee. If the callee is not reached until caller leaves the voice mail server, messages that are shown in the dotted lines will not be executed.

\section{RELATED WORK AND SOLUTION}

Mobility management enables users to roam freely. It is classified into two groups: telecom-based and service-oriented [4]. Telecom based mobility management can be performed in three layers: application layer (e.g. SIP), transport layer (e.g. Stream Control Transmission Protocol) and network layer (Mobile IP V4 and V6, eAll-SIP [10]). Consolidating the Telecom-based and service-oriented approaches, [4] presents a middleware-based approach for mobility management. However, our approach is very similar to the application layer approach; it is specific to real-time communication services.

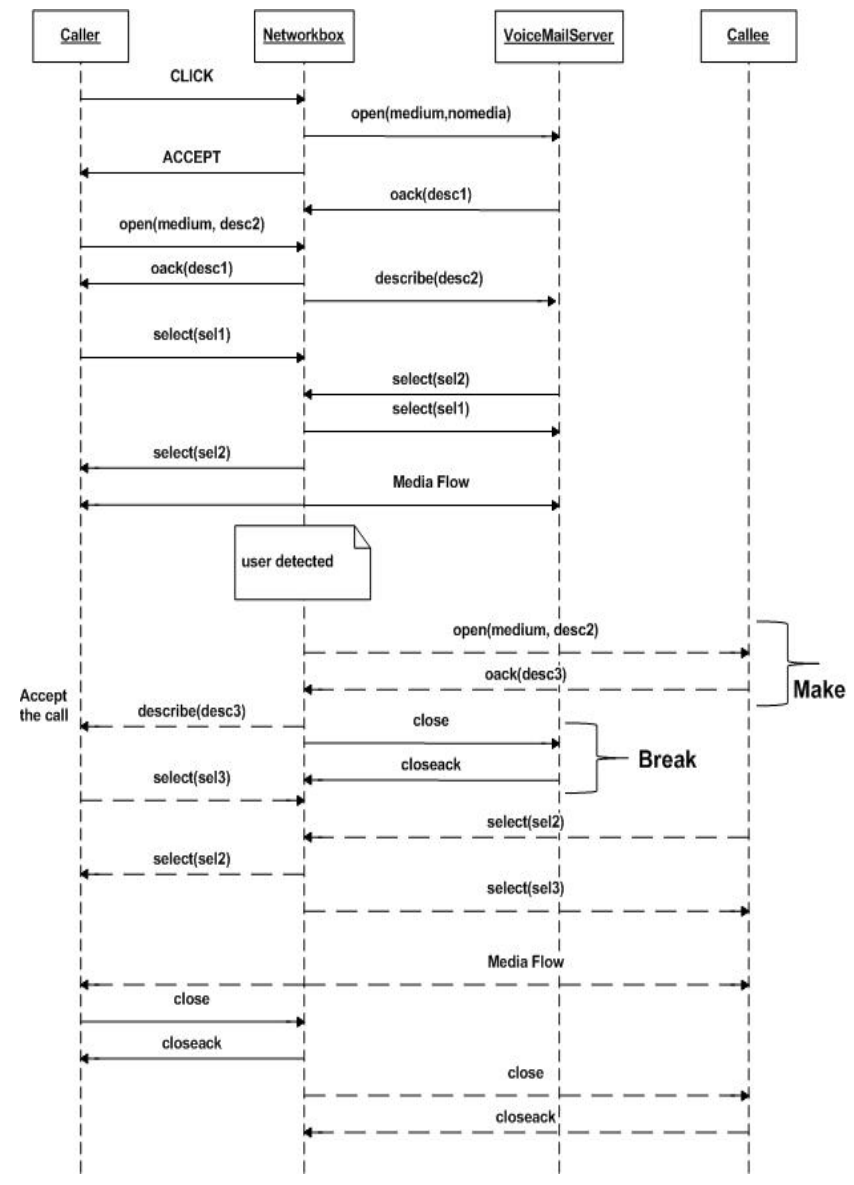

Figure 5: Call flow during the voice mail and call

There are four kinds of mobility (personal mobility [7], terminal mobility, service mobility [8] and session mobility) discussed in the literature [5]. Our work closely involves personal mobility and session mobility. Personal mobility refers to the user's ability to access personalized mobility services that they are subscribed to from anywhere, at any time and using any terminal. Session mobility refers to the user's ability to maintain an active session while moving across networks or switching between terminals. In our proposal, we consider the case where devices are located in the IP network, not in the circuit switched network.

Session transfer across multiple devices can be performed in two ways: partial session mobility [8] and complete session mobility. "Transferring parts of a multimedia session (e.g. video part in one device and audio part in another device) between different devices is defined partial session mobility" 
[8]. Complete session mobility means that signaling and media parts of a session completely transfer from one device to another device. This paper discusses only a complete session transfer.

SIP specification has defined mechanisms related to personal and session mobility. We consider personal mobility at session initiation and session mobility during a session.

\section{A. Personal Mobility at session initiation}

SIP network has a stateful proxy (Forking proxy) [14] that implements three different mechanisms: sequential, parallel and pipeline [11] for personal mobility at the session initiation. This approach pre-requires multiple registrations of user agents to the SIP network and needs to resolve the address of record with different contacts. This service poorly utilizes the resource (many SIP messages over the network) in a parallel search and involves long delay for call set up in a serial search. Integration of knowledge of user mobility is important for delivering this service. The work reported in [11] models the user mobility and derives the values for making the best decision in the forking. The other work related to making a decision in forking is that receiving devices are grouped into active and standby in order to reduce delay and amount of message traffic [12].

In our proposal, context enabler is proposed and is used with network boxes. The network box concerns only two functional interfaces of the context enabler and makes decisions in routing calls at session initiation and during a session.

The articles [13][15][16][19][20] indicate that getting the context information and integrating with communication services is feasible and useful. For example, in [13], using Radio Frequency Identification (RFID), a solution for personal mobility (not session continuity) is proposed for a SIP network that resolves RFID with SIP user identification. In [17] [18], authors propose a special proxy (loosely couple application interaction proxy [17], context-aware SIP proxy [18]) for the SIP network. Even though these proxies are incomplete and more general, they suffer from scalability when many users access the proxy simultaneously.

\section{B. Session Mobility During a session}

Complete session mobility can be performed by either a user element (UE) or a network entity.

1) UE- initiated transfer. REFER method [9] is proposed to transfer calls from one device to another device by an action of the user. The user should know the identity of the target UE and make the transfer using REFER method and Refer-To header. This method allows moving signaling and media paths together and has join and replace headers. The REFER mechanism is hostile to ASs in the network. It means that ASs in old and new signalling paths are different.

This method does not improve the user experience since his involvement is required in knowing the target device addresses and performing the transfer action.

In UE-initiated transfer in SIP, all the devices use a dedicated Address-of-Record (AOR), such as sip:user1@example.com. In our use case, users log in into the system using a single AOR (username). Therefore, mid-call session transfer gives an undesirable result because initiator sends a REFER message with the same AOR for REFER method and contact header. Alternatively, if instances for same AOR can be differentiated using terminal contact or GRUU [25], it is easy to perform the session mobility.

2) Network-Initiated Inter UE session transfer. IMS network deploys SIP ASs for initiating and managing sessions across many devices. In this case, one SIP AS behaves as back-to-back user agent (B2BUA) or executes third party call control by sitting in the signaling path. This approach relieves users from dealing with session transfer. Moreover, networkbased application is easy to integrate with other services such as context information (presence, location) in order to provide rich communication services. However, scalability is a showstopper in the B2BUA approach. It means that single application can not serve for all the users who can access simultaneously. In our approach, each callee has his own network box that can manage the mobility.

In addition, B2BUA manages the session transfer based on SIP addresses. For our proposed use case, a single user logs in from different user agents using the same user ID. Therefore, B2BUA applications have to depend on the forking functionality from the SIP network. Due to this dependency, a B2BUA application should close the session; then, it should restart the session during the session transfer, so a hard handoff. Otherwise, B2BUA should depend on terminal contact or GRUU to support the soft hand-off.

\section{Alternative Solution}

As we discussed earlier in the related work section, solutions for personal and session mobility are working separately. In fact, current IMS specification does not provide clear design guidelines for our use case and as a consequence we have to develop a workaround solution for the IMS architecture. A possible alternative solution is to implement the logic for personal and session mobility is in B2BUA of IMS. It means that B2BUA is able to do forking and (or) session continuity.

We assume that each user in the network has a unique B2BUA application that implements the personal and session mobility. Therefore, this solution is free of scalability problem like our approach.

In this alternative solution, S-CSCF in the IMS architecture should scan the incoming session request (e.g. INVITE) messages and forward to the right B2BUA applications. It means that at least B2BUA application, and S-CSCF is required. In this case, S-CSCF should process at least six SIP messages for address binding and message routing - three (INVITE/ACK/OK) messages between B2BUA and callee, B2BUA and caller. In addition, S-CSCF can be stateful (depending on the implementation) and well-defined routing decisions should be implemented in the IMS network. As indicated in [23], each transaction stateful request requires additional memory, and CPU cycles compared to a stateless transaction request.

As explained in the previous paragraph, this alternative solution suffers from inefficient SIP routing or going through 
S-CSCF to reach the B2BUA application. In our proposal, we eliminate the overhead (delay and processing power, etc) created by S-CSCF and do not consider routing decisions, depending instead on simple HTTP. In addition, our call flow in Figure 4 reduces session set-up time and session transfer time (session mobility) to compare to 3PCC call flow [22]. Due to space constraint, we leave this analysis to interested readers.

Apart from the alternative solution, there are many proposals (for example [24]) for improving the scalability in the IMS architecture. These proposals are typically loadbalancing techniques and resources management at infrastructure and services levels. Our solution - an individual network box for each callee in the Web-based communication system is very simple compared to the existing proposals which need more computing resources and engineering efforts.

\section{CONCLUSION}

We have proposed a solution for user mobility that is realized in a Web-based communication system. The solution consists of a descriptive model that simplifies the architecture when a user logs in from many terminals. In the proposed model, a network box performs as forking proxy, B2BUA and registration server. Our solution focuses on piecewise protocols that are connected in the network box. Moreover, we provide solutions for two different use cases such as networkinitiated session transfer, and missed call situation (sessionbased composition).

For the scalability problem, our solution depends on the HTTP for routing at the infrastructure level; it depends on an individual network box in the Web-based communication system at the service level; our solution is scalable vertically. From our simple solution, we conclude that our solution saves the computing processing power at least by removing six SIP messages parsing and processing overhead per session in a SIP proxy.

We have identified two future research paths. First, we will implement the proposed solution in order to compare performance metrics such as CPU needed in the Web server (hosting for the network box) and SIP server (B2BUA). This implementation work enables us to quantify the latency in the session setup/transfer conditions. Second, we will extend the proposed model for supporting the network-initiated partial session transfer over multiple devices that are situated around a user.

\section{REFERENCES}

[1] Zave, P. and Cheung, E. 2006. Compositional control of IP media. In Proceedings of the 2006 ACM CoNEXT Conference(Lisboa, Portugal, December 04 - 07, 2006). CoNEXT '06. ACM, New York, NY, 1-12. DOI= http://doi.acm.org/10.1145/1368436.1368461.

[2] The Web Socket API, http://dev.w3.org/html5/websockets/

[3] The Web Socket protocol, http://tools.ietf.org/html/draft-hixiethewebsocketprotocol-74
[4] Rong, L., Fredj, M., Issarny, V., and Georgantas, N. 2007. Mobility management in B3G networks: a middleware-based approach. In international Workshop on Engineering of Software Services For Pervasive Environments: in Conjunction with the 6th ESEC/FSE Joint Meeting (Dubrovnik, Croatia, September 04 - 04, 2007). ESSPE '07. ACM, New York, NY, 41-45.

[5] Hasswa, A., Hassanein, H., and Taha, A. M. Facilitating 4G convergence using IMS. In Proceedings of the 2009 Conference on information Science, Technology and Applications (Kuwait, Kuwait, March 20 - 22, 2009). ISTA '09. ACM, New York, NY, 59-66.

[6] F. Vakil, et al, "Supporting Service Mobility with SIP", IETF, Dec. 2000

[7] H. Schulzrinne, "Personal mobility for multimedia services in th Workshop on Interactive Distributed Multimedia Systems and Services (IDMS, Mar. 1996

[8] Tuijn, J. A. and Bijwaard, D. 2008. Spanning a multimedia session across multiple devices. Bell Lab. Tech. J. 12, 4 (Feb. 2008), 179-193.

[9] R;Sparks, "The Session Initiation Protocol (SIP) Refer Method", RFC 3515, IETF,Apr,2003

[10] H. Miyajima, L. Zhang, H. Hayashi, and T. Fujii, "An Implementation of Enhanced All-SIP Mobility," Proc. of PIMRC2008, Sep. 2008.

[11] Tsan-Pin Wang and KauLin Chiu, "An Efficient Scheme for Supporting Personal Mobility in SIP-based VoIP Services," IEICE Trans. on Communications (Special Section on Mobile Multimedia Communications), Vol. 89-B, No.10, pp.2706-2714, Oct. 2006. (SCI)

[12] T. P. Wang, H. Y. Lee, "User Location Management for Personal Mobility in SIP-based VoIP Services", Third International Conference on Communications and Networking in China, Hangzhou, China, pp. 910-914, August 2008, ISBN: 978-1-4244-2374-3.

[13] Pen-ho Chang and Tsan-Pin Wang, "Design and Implementation of an Integrated RFID and VOIP System for Supporting Personal Mobility," International journal of Computer Networks \& Communications (IJCNC), Vol. 1, No. 3, pp.92-103, Oct. 2009.

[14] J. Rosenberg, H. Schulzrinne, G. Camarillo, A. Johnston, J. Peterson, R. Sparks, M. Handley, E. Schooler, "SIP: Session Initiation Protocol", RFC 3261, June 2002

[15] C-CAST project, http://www.ict-ccast.eu/

[16] B. Moltchanov, M. Knappmeyer, C.A. Licciardi, N. Baker, "ContextAware Content Sharing and Casting", 12th ICIN, Bordeaux, France, October 2008

[17] Jacob, C.; Pfeffer, H.; Linner, D.; Steglich, S.; Li Yan; Ma Qifeng; , "Automatic routing of semantic SIP messages in IMS," Internet Multimedia Services Architecture and Applications, 2008. IMSAA 2008. 2nd International Conference on, vol., no., pp.1-6, 10-12 Dec. 2008 doi: 10.1109/IMSAA.2008.4753908

[18] Tang Tang, Zhengkun Mi, Rongqun Peng, "Adaptive Service Provisioning through Context-Aware SIP Proxy," icns, pp.277-281, Fourth International Conference on Networking and Services (icns 2008), 2008

[19] Barachi, M.; Kadiwal, A.; Glitho, R.; Khendek, F.; Dssouli, R.; , "The design and implementation of architectural components for the integration of the IP multimedia subsystem and wireless sensor networks,"Communications Magazine, IEEE, vol.48, no.4, pp.42-50, April2010

[20] A. Gluhak et al. "e-SENSE Reference Model for Sensor Networks in B3G Mobile Communication Systems", Information society technologies (IST) 2006.

[21] Boussard, Mathieu; Jabaud, Philippe; Le Berre, Olivier; Poussiere, Fabrice; Labrogere, Paul, "Communication hyperlinks: Call me my way," Intelligence in Next Generation Networks, 2009. ICIN 2009. 13th International Conference on, vol., no., pp.1-5, 26-29 Oct. 2009

[22] Shanmugalingam, Sivasothy; Crespi, Noel; Labrogere, Paul; "My Own Communication Service Provider", International Conference on Ultra Modern Telecommunications, 2010. ICUMT'10, Oct. 2010.

[23] Cortes, M.; Esteban, J.O.; Jun, H.; , "ISE03-3: Towards Stateless Core: Improving SIP Proxy Scalability," Global Telecommunications Conference, 2006. GLOBECOM '06. IEEE, vol., no., pp.1-6, Nov. 27 2006-Dec.

[24] Bellavista, P.; Corradi, A.; Foschini, L.; , "Understanding and enhancing the scalability of IMS-based services for Wireless Local Networks," Local Computer Networks, 2009. LCN 2009. IEEE 34th Conference on, vol., no., pp.1033-1039, 20-23 Oct. 2009.

[25] J. Rosenberg, "Obtaining and Using Globally Routable User Agent URIs (GRUUs) in the Session Initiation Protocol ", RFC 5627, IETF,Oct,2009 